\title{
The Critical View of Safety : une attitude pour prévenir l'erreur d'identification dans les cholécystectomies laparoscopiques.
}

\section{The Critical View of Safety : an attitude to prevent misidentification during laparoscopic cholecystectomy.}

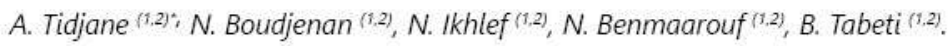

(1) - Service de chirurgie hépatobiliaire et greffe du foie, EHU, 1er Novembre 1954, Oran.

(2) - Département de Médecine, Faculté de médecine, Université Oran1, Oran.

Adresse : Service de chirurgie hépatobiliaire et greffe du foie, Etablissement Hospitalier Universitaire d'Oran 1er Novembre 1954 , BP N4166 Ibn Rochd, Oran.

Email : tidjane.anisse@univ-oran1.dz

Déclarations de liens d'intérêts : Les auteurs de cet article déclarent n'avoir aucun lien d'intérêt.

\section{Résumé}

Avec la généralisation de la cholécystectomie laparoscopique, l'incidence des traumatismes opératoires des voies biliaires a augmentée de façon significative. La perte de la sensation haptique, la vision bidimensionnelle, et l'éclairage centré favorisent l'illusion aboutissant à des erreurs d'identifications, où le chirurgien confond voie biliaire principale et canal cystique. Ce phénomène explique l'augmentation des traumatismes biliaires à l'ère de la laparoscopie.

Récemment, et grâce aux recommandations des sociétés savantes comme la SAGES sur les bonnes pratiques chirurgicales, l'incidence de ces traumatismes est en régression et tend à rejoindre celle décrite à l'ère de la chirurgie ouverte. Parmi ses recommandations, la SAGES met l'accent sur l'adoption par tous les chirurgiens de la "Critical View of Safety" durant la réalisation de la cholécystectomie par voie laparoscopique. L'objectif de cette mise au point est de décrire cette attitude, mais surtout analyser son efficacité et ses limites dans la prévention des traumatismes opératoires des voies biliaires.

Mots clés Vésicule biliaire, Bonne pratique, Coelioscopie, Prévention, Plaie biliaire, Cholangiographie.

\begin{abstract}
With the generalization of laparoscopic cholecystectomy, the incidence of operative bile ducts injury has increased significantly, the loss of haptic sensation, the two-dimensional vision, and the centered lighting favor the illusion leading to misidentifications, as consequences the surgeon confuses the main bile duct and the cystic duct. This phenomenon explains this increase in the incidence of bile duct injuries in the era of laparoscopy.

Recently, and thanks to the recommendations of somelearned societies such as SAGES on good surgical practices, the incidence of these complications is declining and tends to join that described in the era of open surgery. Among its many recommendations, SAGES recommends the adoption by all surgeons of the "Critical View of Safety" during the performance of laparoscopic cholecystectomy, an attitude that we will describe, and analyze its effectiveness and its limits in prevention of operative bile ducts injury.
\end{abstract}

Key words Gallbladder, Best practice, Laparoscopy, Prevention, Bile duct injury, Cholangiography.

Ju i n. 2021 36




\section{Introduction}

Le traumatisme opératoire des voies biliaires (TOVB) est un accident grave, qui survient souvent durant la réalisation d'une cholécystectomie. Avec l'introduction de la chirurgie laparoscopique, l'incidence des TOVB a augmenté de façon significative en comparaison avec l'incidence de cette complication à l'ère de la chirurgie classique. Plusieurs facteurs semblent participer à cette recrudescence des TOVB : La courbe d'apprentissage, l'usage de certains instrument dangereux, mais la principale cause est l'illusion avec erreur d'identification des éléments anatomiques aboutissant à la confusion entre le canal cystique et la voie biliaire principale ${ }^{[1,2]}$. Cette erreur d'identification propre à la chirurgie laparoscopique aboutit à des lésions biliaires et vasculaires graves qui, non reconnues et traitées à temps, peuvent être fatales pour le patient ${ }^{[3.5]}$.

La courbe d'apprentissage et l'introduction de nouvelles recommandations techniques par les sociétés savantes avaient permis de réduire de façon spectaculaire l'incidence des TOVB provoqués durant la cholécystectomie laparoscopique $(\mathrm{CL})$. Cependant, la confusion demeure le principal facteur responsable de la survenue des TOVB.

En 1996, Strasberg proposait l'adoption par les chirurgiens d'une attitude qui permettrait, selon son promoteur, de pallier à ce grave problème. Cette technique est nommée communément The Critical View of Safety (CVS) ${ }^{[6]}$. L'objectif de notre mise au point est de décrire cette technique et d'évaluer son efficacité à travers les différentes expériences publiées.

\section{Technique de réalisation de la CVS}

Pour l'achèvement de la CVS, trois conditions doivent être réunies lors de l'abord de la vésicule biliaire par voie rétrograde laparoscopique :

- La dissection du triangle de Calot : le triangle de Calot devra être disséqué de la graisse et du tissu fibreux l'enveloppant, la voie biliaire n'est pas obligatoirement disséquée. Le triangle de Calot est l'espace compris entre le canal cystique et l'artère cystique, et est délimité en dedans par la voie biliaire principale et en dehors par la vésicule biliaire.

Pour réaliser cette condition, une bonne exposition du triangle de Calot est nécessaire, cette exposition n'est possible que si le fond de la vésicule biliaire est tiré vers le haut et en dehors, et en même temps l'infundibulum est dégagé en bas et en dehors et ce durant tout le temps de la dissection. II est clair donc que l'utilisation de quatre trocarts durant la $\mathrm{CL}$ est une obligation pour assurer cette étape sans incidents ${ }^{[6.7]}$.

- La libération de la vésicule biliaire du $1 / 3$ inférieur de son lit : La vésicule biliaire devra être totalement libérée de son lit dans son $1 / 3$ inférieur, et sur ses deux faces antérieure et postérieure. Une fois réalisée, cette libération permettra la création d'une large fenêtre pouvant laisser passer de travers, sans aucune difficulté les instruments de dissection (Figure 1).
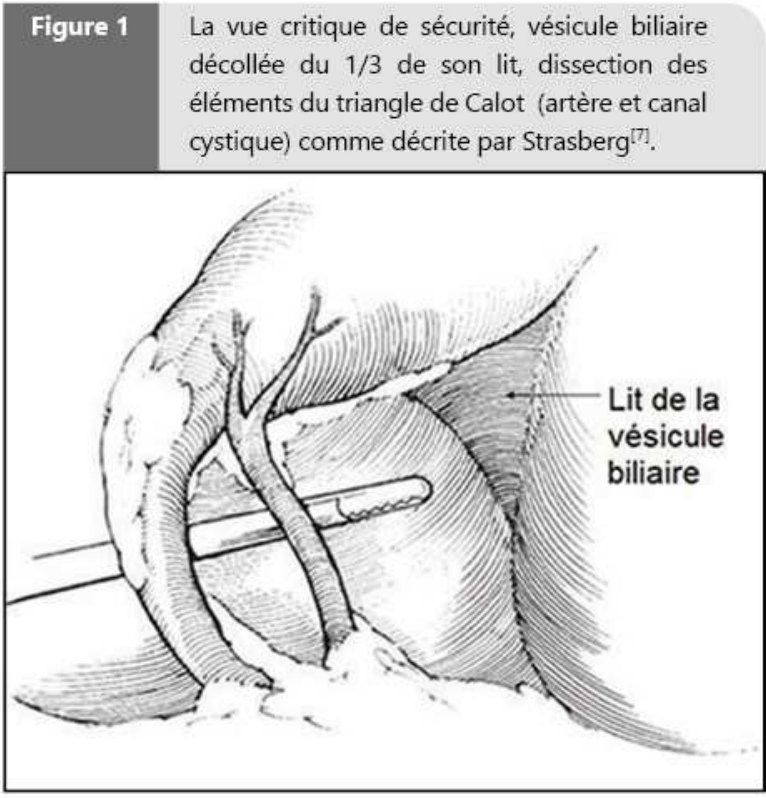

- La vue critique : A cette étape, la vésicule biliaire est presque totalement libérée et reste attachée par son fond au foie, et par son artère et son canal cystique au pédicule hépatique. Le chirurgien devra tout en déplaçant l'infundibulum de la vésicule biliaire en avant et en haut puis en bas, de façon répétée, s'assurer que seules deux structures relient la vésicule biliaire au pédicule hépatique. C'est en ce temps crucial que le chirurgien remet en cause l'identité des éléments disséqués et remet en cause ce qui est censé être acquis, ce qui est sensé être le canal cystique et l'artère cystique (Figure 2).

Devant l'impossibilité d'obtenir cette CVS, il est prudent de vérifier l'anatomie biliaire par des techniques d'imageries peropératoires, entre autre une cholangiographie peropératoire ${ }^{[6]}$.

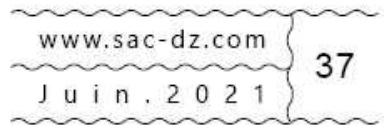




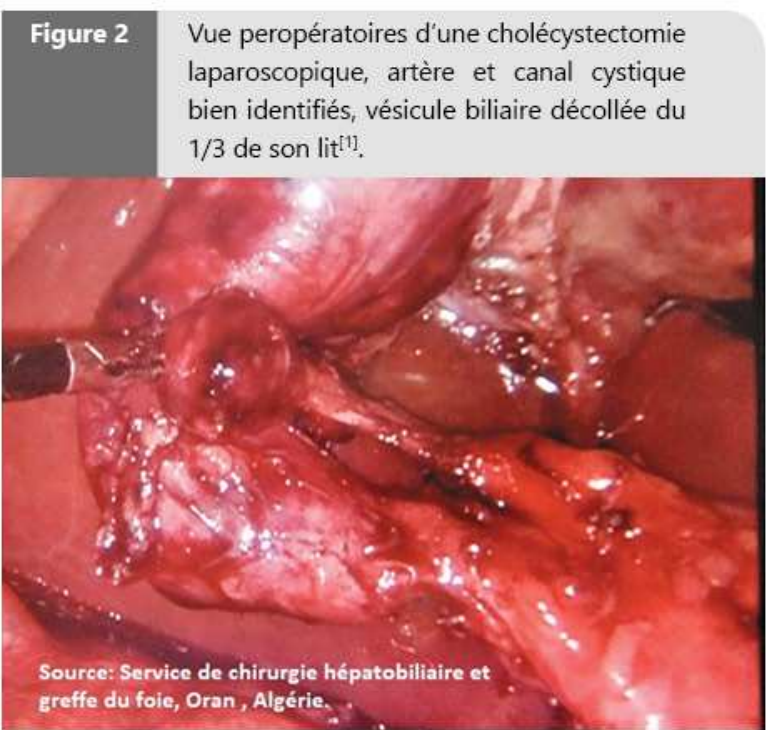

A la fin, l'artère cystique est liée par des clips et sectionnée du côté le plus proche de la vésicule biliaire, et le canal cystique est à son tour lié et sectionné de la même manière mais en restant à une distance raisonnablement éloignée de ce qui est considérée comme lieu de jonction cysticocholédocienne ${ }^{[7]}$. A la fin, un examen soigneux du champ opératoire permet de revoir les emplacements des clips ou des ligatures et d'explorer le lit de la vésicule biliaire afin de détecter toute fuite biliaire. L'examen de la pièce définitive sur la table opératoire permet de vérifier une fois encore les éléments anatomiques coupés ${ }^{[6]}$.

\section{Efficacité de la CVS}

L'adoption de la CVS avait permis d'obtenir une diminution significative des TOVB provoqués par laparoscopie. Malgré la large diffusion de cette technique de sécurité, les plaies biliaires continuent à se produire, les questions qui se posaient, étaient :

- Les chirurgiens qui déclarent respecter la CVS le font-t-ils vraiment? Respectent-t-ils les 3 phases de cette technique?

- En cas d'impossibilité d'avoir cette vue critique, que devrait faire le chirurgien?

Avgerinos et al. rapportent dans une série mono-centrique de $1000 \mathrm{CL}$ un respect de la CVS de l'ordre de 95,4\%, le respect de la CVS était prouvé par prise de photos durant la chirurgie. Dans cette série aucun TOVB n'était déclaré ${ }^{[8]}$. Une autre étude rétrospective mono-centrique de Nijssen et al. sur 1108 cholécystectomies réalisées entre 2009 et 2011, rapportait 1,7\% de TOVB. Les chirurgiens déclaraient respecter la vue critique dans $80 \%$ des cas, mais finalement après contrôle des enregistrements vidéos des interventions chirurgicales réalisées, il s'avère que dans seulement $18,7 \%$ des CL la CVS était réellement respectée, et quand cette technique était respectée aucune plaie biliaire n'était provoquée ${ }^{[9]}$

D'autres expériences rapportent des résultats aussi positifs, comme ceux de Sanjay et al. avec $0 \%$ de plaies biliaires et un respect de $87 \%$ de la CVS durant $447 \mathrm{CL}$ dont $40 \%$ réalisés chez des patients en cholécystite aigue. Dans son expérience, l'impossibilité d'avoir la CVS était une indication immédiate à la conversion en chirurgie classique sans aucune hésitation ${ }^{[10]}$, cette attitude bien que à notre avis semble exagérée, semble toutefois justifiée devant l'impossibilité d'obtenir une cholangiographie peropératoire avec une imagerie interprétable.

Les études analysant les résultats de la CVS sont résumées dans le tableau suivant (Tableau 1).

Tableau 1 Résultats de l'adoption de la CVS

\begin{tabular}{|l|c|c|c|}
\hline Auteur & Nombre de cas & Taux de respect de la CVS & Pourcentage des traumatismes biliaires \\
\hline Heisterman et al. 2006 & 100 & $97 \%$ & $1,6 \%$ \\
\hline Avgerinos et al. 2009 ${ }^{[8]}$ & 1000 & $95,4 \%$ & $0 \%$ \\
\hline Yegiyants et Collins. 2008 ${ }^{[12]}$ & 3042 & Non évalué & $0,03 \%$ \\
\hline Sanjay et al. 2010 & $\begin{array}{c}{[10]} \\
\text { cholécystites aigues) }\end{array}$ & $110 \%$ & $0 \%$ \\
\hline Nijssen et al. 2015 & $108 \%$ & $10,8 \%$ & $1,7 \%(0 \%$ quand la CVS est respectée $)$ \\
\hline Tsalis et al. 2015 & 929 & $95,8 \%$ & $0 \%$ \\
\hline Kaya et al. 2017 & 120 & $100 \%$ & $0 \%$ \\
\hline
\end{tabular}

suin.


Comme pour la $\mathrm{CL}$, cette technique préventive nécessite une courbe d'apprentissage. Ainsi, Chen et al. rapportent une efficacité de l'enseignement de cette technique après la réalisation de la $50^{\text {ème }} \mathrm{CL}$. Selon les auteurs, les 43 résidents en chirurgie ayant participé pour élaborer cette étude, amélioraient leurs reconnaissances des éléments clés de la CVS après un enseignement vidéo de plus de 50 $\mathrm{CL}$. L'auteur conclut que la CVS nécessite un enseignement pratique, et que plus le chirurgien prend l'habitude à la réaliser, plus il apprend à respecter ses étapes clés avec perfection ${ }^{[15]}$.

Nakazato et al. constatent qu'après enseignement vidéo de la CVS, les douze chirurgiens inclus dans l'étude amélioraient de façon significative le taux de réussite de la réalisation de la CVS. L'auteur conclut que l'enseignement continu de la CVS permettrait d'augmenter la fréquence et surtout la qualité de réalisation de la CVS dans la pratique quotidienne des chirurgiens lors de la réalisation de $\mathrm{CL}^{[16]}$.

\section{La CVS et les autres techniques d'identification}

D'autres techniques d'identification des éléments anatomiques durant la réalisation de la cholécystectomie laparoscopique ont été décrites et comparées à la CVS. Nous citons à titre d'exemple: la systématisation de la cholangiographie peropératoire, la technique infundibulaire, la dissection de la voie biliaire principale et la cholécystectomie antérograde sont des techniques décrites comme étant des techniques d'identifications efficaces des éléments anatomiques lors de la $\mathrm{CL}^{[17]}$. Bien que la CVS soit considérée comme la technique de référence pour prévenir les erreurs d'identification durant cette chirurgie, cette distinction n'est basée que sur des avis d'experts ou des séries de cas, donc à faible niveau de preuve (Niveau 4) ${ }^{[17]}$.

La cholangiographie peropératoire a une place incontournable dans la reconnaissance peropératoire des TOVB et non dans la prévention de ces traumatismes. Car au moment du cathétérisme du canal identifié comme étant le canal cystique, le traumatisme en cas d'erreur aura déjà était causé, il est donc important à notre avis de souligner que la cholangiographie peropératoire est un moyen de détection précoce du TOVB, mais dont l'impact sur le pronostic des TOVB est évident, car un TOVB reconnu immédiatement a un meilleur pronostic qu'un TOVB reconnu en phase de péritonite biliaire ${ }^{[1]}$.

La cholangiographie peropératoire durant la réalisation d'une $\mathrm{CL}$ se heurte à des difficultés, le manque de formation sur la technique de sa réalisation, la non disponibilité permanente de cette imagerie dans tous les centres hospitaliers, mais surtout un manque de formation en matière d'interprétation de la cholangiographie ${ }^{[18]}$. Comme conséquence, le moment venu le chirurgien ne pourra pas réaliser cette imagerie ou n'aura pas les compétences pour l'interpréter convenablement.

Dans un sondage réalisé par nos équipes sur les pratiques de la cholécystectomie en Algérie et ayant touché 122 chirurgiens Algériens exerçant dans 36 wilaya sur les 48 du pays, aussi bien dans des structures publiques que libérales, aucun des chirurgiens participant ne réalise cette technique d'imagerie de façon systématique durant la $\mathrm{CL}$. Trente pourcent des chirurgiens déclarent ne pas disposer de moyens techniques pour pouvoir la réaliser à tout moment, et $2 \%$ avouent ne pas savoir comment réaliser une cholangiographie peropératoire par voie laparoscopique ${ }^{[19]}$.

D'autres techniques d'imagerie non invasive permettant d'obtenir une cartographie biliaire en peropératoire avant la section biliaire sont à prendre en considération. La plus innovante de ces techniques est la cholangiographie fluorescente au vert d'indocyanine. Cette technique consiste en l'injection par voie intraveineuse d'une dose de vert d'indocyanine, cette substance est métabolisée et excrétée par le foie dans les voies biliaires, à l'excitation de cette substance par une lumière polarisée, les voies biliaires deviennent visibles permettant ainside bien voir la voie biliaire principale et le canal cystique. Dans une publication de Daskalakis et al sur une série de 184 cholécystectomies réalisée par Robot, la voie biliaire principale était clairement visible dans $94 \%$ des $\operatorname{cas}^{[20]}$. D'autres publications rapportent des résultats mono-centriques encourageants avec une réduction de l'incidence des traumatismes majeurs des voies biliaires après utilisation de cette technique durant la $\mathrm{CL}^{[21]}$

\section{Conclusion}

The Critical View of Safety est une attitude qui permet de prévenir l'erreur d'identification responsable de la majorité des traumatismes opératoires des voies biliaires survenant durant la réalisation d'une cholécystectomie laparoscopique. Cette technique devra faire partie intégrante de l'enseignement pratique de la cholécystectomie laparoscopique. Le chirurgien en fin de formation devra obligatoirement savoir comment réaliser la Critical View of Safety, et en cas de difficulté pouvoir réaliser une cholangiographie peropératoire par voie laparoscopique et savoir interpréter correctement cette imagerie.

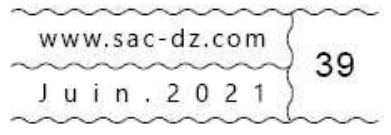




\section{Références}

[01]- Tidjane A. Management of operative bile duct injury in the region of Oran. [Internet] [Thesis]. [Oran, Algeria] : University Oran 1; 2019. Available from : https://theses. univ-oran1.dz/document/1012019003t.pdf

[02]- Davidoff AM, Pappas TN, Murray EA, Hilleren DJ, Johnson $R D$, Baker $M E$, et al. Mechanisms of major biliary injury during laparoscopic cholecystectomy. Ann Surg. 1992 Mar;215(3):196-202.

[03]- Hogan NM, Dorcaratto D, Hogan AM, Nasirawan F,

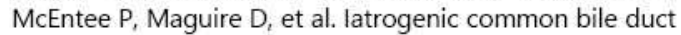
injuries: Increasing complexity in the laparoscopic era: A prospective cohort study. International Journal of Surgery. 2016 Sep 1;33:151-6.

[04]- Strasberg SM, Helton WS. An analytical review of vasculobiliary injury in laparoscopic and open cholecystectomy. Hpb. 2011;13(1):1-14.

[05]- Tidjane A, Boudjenan Serradj N, Ikhlef N, Benmaarouf $\mathrm{N}$, Tabeti B. Factors influencing the occurrence of biliary stricture above the confluence in major bile ducts injuries : Analysis of a case series. Annals of Medicine and Surgery. 2020 Sep 1;57:334-8.

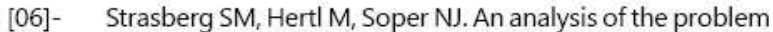
of biliary injury during laparoscopic cholecystectomy. J Am Coll Surg. 1995 Jan;180(1):101-25.

[07]- Strasberg SM, Brunt LM. Rationale and Use of the Critical View of Safety in Laparoscopic Cholecystectomy. Journal of the American College of Surgeons. 2010 Jul 1;211(1):132-8.

[08]- Avgerinos C, Kelgiorgi D, Touloumis Z, Baltatzi L, Dervenis C. One Thousand Laparoscopic Cholecystectomies in a Single Surgical Unit Using the "Critical View of Safety" Technique. J Gastrointest Surg. 2009 Mar 1;13(3): 498-503.

[09]- Nijssen M a. J, Schreinemakers JMJ, Meyer Z, van der Schelling GP, Crolla RMPH, Rijken AM. Complications After Laparoscopic Cholecystectomy: A Video Evaluation Study of Whether the Critical View of Safety was Reached. World J Surg. 2015 Jul;39(7):1798-803.

[10]- Sanjay P, Fulke JL, Exon DJ. 'Critical View of Safety' as an Alternative to Routine Intraoperative Cholangiography During Laparoscopic Cholecystectomy for Acute Biliary Pathology. J Gastrointest Surg. 2010 Aug 1;14(8):1280-4.

[11]- Heistermann HP, Tobusch A, Palmes D. [Prevention of bile duct injuries after laparoscopic cholecystectomy. 'The critical view of safety']. Zentralbl Chir. 2006 Dec;131(6):460-5.
[12]- Yegiyants S, Collins JC. Operative strategy can reduce the incidence of major bile duct injury in laparoscopic cholecystectomy. Am Surg. 2008 Oct; 74(10):985-7.

[13]- Tsalis K, Antoniou N, Koukouritaki Z, Patridas D, Christoforidis E, Lazaridis C. Open-access Technique and "Critical View of Safety" as the Safest Way to Perform Laparoscopic Cholecystectomy. Surgical Laparoscopy, Endoscopy \& Percutaneous Techniques. 2015 Apr $1 ; 25(2): 119-24$

[14]- Kaya B, Fersahoglu MM, Kilic F, Onur E, Memisoglu K. Importance of critical view of safety in laparoscopic cholecystectomy: a survey of 120 serial patients, with no incidence of complications. Ann Hepatobiliary Pancreat Surg. 2017 Feb;21(1):17-20.

[15]- Chen CB, Palazzo F, Doane SM, Winter JM, Lavu H Chojnacki KA, et al. Increasing resident utilization and recognition of the critical view of safety during laparoscopic cholecystectomy: a pilot study from an academic medical center. Surg Endosc. 2017 Apr 1;31(4):1627-35.

[16]- Nakazato T, Su B, Novak S, Deal S, Kuchta K, Ujiki M. Improving attainment of the critical view of safety during laparoscopic cholecystectomy. Surgical Endoscopy. 2020 Sep 1;34(9):4115-23.

[17]- Strasberg SM, Brunt LM. The Critical View of Safety: Why lt Is Not the Only Method of Ductal Identification Within the Standard of Care in Laparoscopic Cholecystectomy. Annals of Surgery. 2017 Mar;265(3): 464.

[18]- Sanjay P, Tagolao S, Dirkzwager I, Bartlett A. A survey of the accuracy of interpretation of intraoperative cholangiograms. HPB (Oxford). 2012 Oct;14(10):673-6.

[19]- Tidjane A, Boudjenan Serradj N, Ikhlef N, Benilha S, Benmaarouf N, Tabeti B. Surgical practices during cholecystectomy in Algeria: results of a survey. International Surgery Journal. 2020 Mar 26:7(4):981-5.

[20]- Daskalaki D, Fernandes E, Wang X, Bianco FM, Elli EF, Ayloo $S$, et al. Indocyanine green (ICG) fluorescent cholangiography during robotic cholecystectomy: results of 184 consecutive cases in a single institution. Surgical innovation. 2014;21(6):615-621.

[21]- Broderick RC, Lee AM, Cheverie JN, Zhao B, Blitzer RR, Patel RJ, et al. Fluorescent cholangiography significantly improves patient outcomes for laparoscopic cholecystectomy. Surg Endosc. 2020 Oct 14;1-11. 